\title{
Periodic limb movement disorder and restless legs syndrome
}

\section{De Volder \\ Dept of Psychiatry University Hospital Antwerp 2650 Antwerp Belgium E-mail:ilse.de.volder@uza.be}

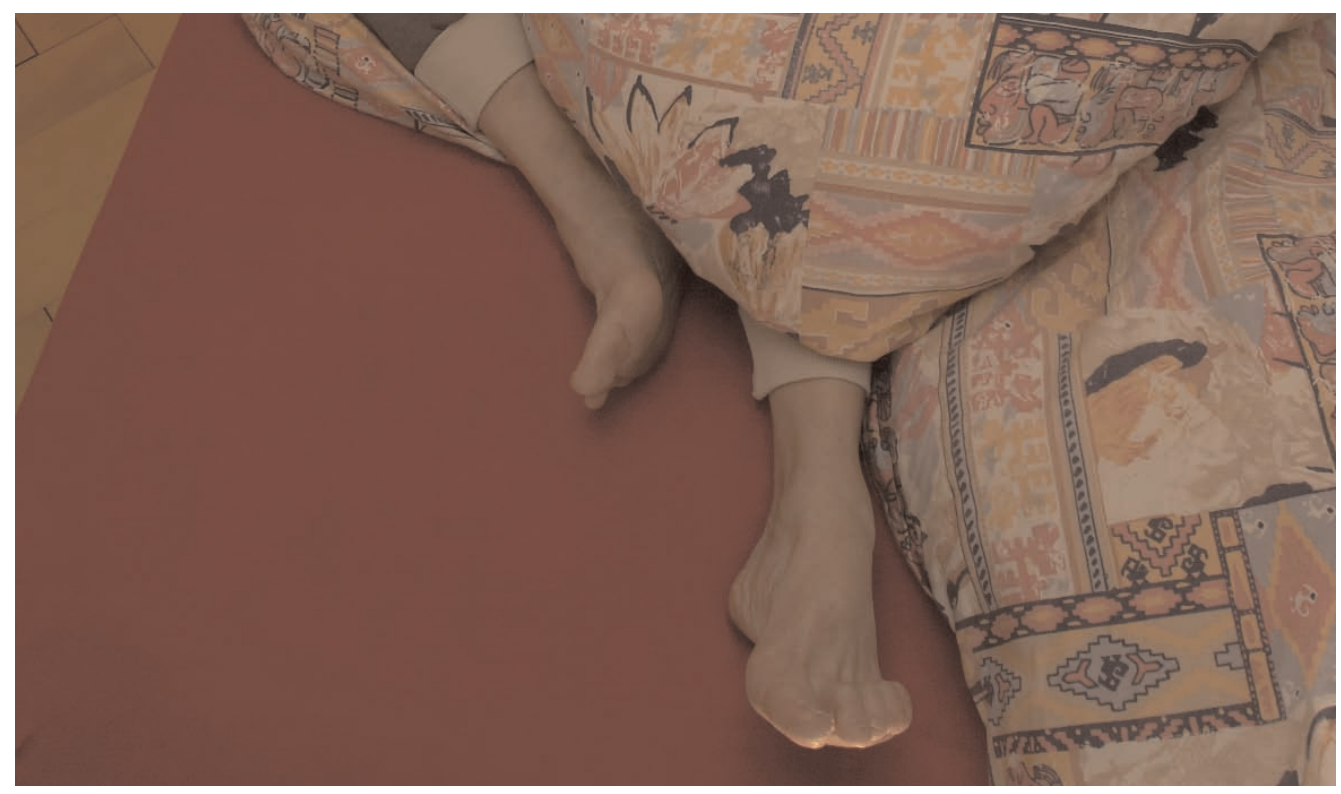

Restless legs syndrome (RLS) is a clinically defined syndrome, where the patient has a desire to move their legs due to strange and unpleasant feelings in the limbs. As the symptoms are very vague, many patients remain undiagnosed. Some of the issues relating to RLS are presented here.

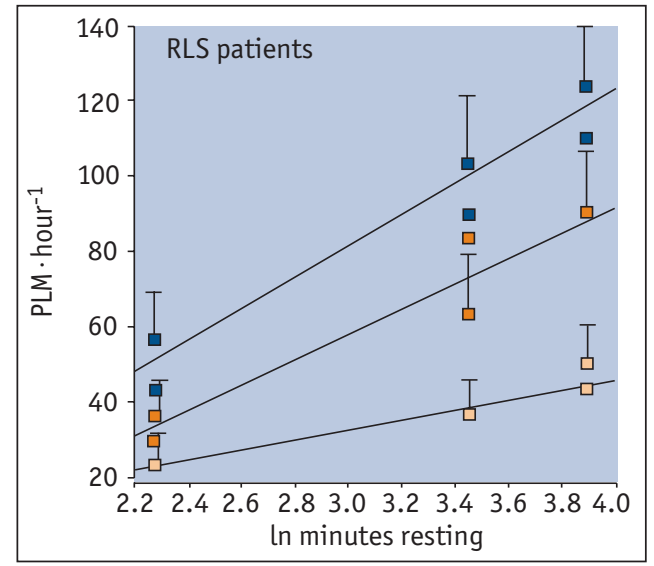

Figure 1

Circadian aspects of RLS symptomatology. Reproduced with permission from Allen et al. 2005.
The clinical definitions for RLS were only established in 1995, when research on this disorder began. RLS was defined and described as the following: 1) a desire to move the limbs, which is often associated with paraesthesiae and dysaesthesiae; 2) having symptoms that are worse or present only during rest and are partially or temporarily relieved by activity; 3) having motor restlessness; and 4) experiencing nocturnal worsening of symptoms. In 2004, the definitions of RLS were updated. Subsequently, RLS was defined as an urge to move the legs, accompanied by unpleasant or uncomfortable sensations in the legs, which begin or worsen during periods of rest or inactivity, such as lying or sitting down, and are worse during the evening or night than during the day or only occur during 
the night (figure 1). These sensations are partially or totally removed by movements such as walking or stretching.

As the symptoms of this disorder are quite vague, many patients remain undiagnosed and untreated, causing serious morbidity. The prevalence in Europe is $8-15 \%$ and around 1-3\% in Asia.

The International Restless Legs Scale is a validated questionnaire, which is used for rating severity and is very useful in a clinical setting. A second method that has recently been used to evaluate the degree of RLS is the suggested immobilisation test (SIT). This method allows quantification of leg movements from the right and left anterior tibialis muscle surface electromyogram during a 1-hour period of immobilisation prior to bedtime. The SIT has been validated in a small sample of patients and controls, and was found to have a high sensitivity and specificity for diagnosing RLS. Recently, a leg discomfort scale was added to the original SIT procedure, which was administered every 5 minutes during the SIT in order to evaluate the sensory component of RLS. However, the sensitivity and specificity of this method have not yet been assessed. The SIT can be used in an assessment of severity, but is not needed for confirmation of a diagnosis.

RLS has been found to have an association with pregnancy, renal failure, anaemia, neuropa- thy, attention deficit hyperactivity disorder and the use of some medications, such as neuroleptics and antidepressants.

RLS is a diagnosis of the awake state, and is often associated with periodic limb movement disorder (PLMD), which is a phenomenon of sleep. PLMD is characterised by involuntary leg twitching or jerking movements during sleep that typically occur every 15-40 seconds, sometimes throughout the night. These symptoms can cause repeated awakenings or microarousals and severely disrupted sleep, leading to insomnia or hypersomnia during the day.

Both RLS and PLMD are thought to share the same pathogenesis, which has yet to be fully elucidated. RLS patients may have low plasma and CSF ferritin levels, and a dysfunctional blood brain barrier, resulting in disrupted transport of ferritin and iron, thus resulting in low availability of iron in the brain. A dopaminergic dysfunction in the striatum of the brain is also believed to play a role in the pathogenesis of RLS.

RLS often runs in families and, hence, a hereditary factor is likely. The first RLS gene has recently been described.

RLS and PLMD share the same therapy. Dopaminergic treatment and clonazepam are frequently used. Gabapentin is also an option. Opioids, e.g. subcutaneous apomorphine, are reserved for more severe cases.

\section{Suggested further reading}

Sleep Medicine. Vol. 3. Suppl. 1. Elsevier, 2002.

A whole volume dedicated to RLS and PLMD, with interesting articles on genetics, epidemiology and therapy.

Walters AS. Toward a better definition of the restless legs syndrome. The International Restless Legs Syndrome Study Group. Mov Disord 1995; 10: 634-642.

Allen RP, Earley CJ. Restless legs syndrome: a review of clinical and pathophysiologic features. J Clin Neurophysiol 2001; 18: 128-147.

Manconi M, Govoni V, De Vito A, et al. Restless legs syndrome and pregnancy. Neurology 2004; 63: 1065-1069.

Krieger J, Schroeder C. Iron brain and restless legs syndrome. In: Sleep Medicine Reviews. Vol. 5. 2001; pp. 277-289. Comprehensive review on the basics of iron metabolism.

Desautels A, Turecki G, Montplaisir J. Restless legs syndrome: confirmation of linkage to chromosome 12q, genetic heterogeneity, and evidence of complexity. Arch Neurol 2005; 62: 591-596.

Schapira AH. Restless legs syndrome: an update on treatment option. Drugs 2004; 64: 149-158.

Lesage S, Hening WA. The restless legs syndrome and periodic limb movement disorder: a review of management. Semin Neurol 2004; 24: 249-259.

Standards of Practice Committee of the American Academy of Sleep Medicine. Practice parameters for the dopaminergic treatment of restless legs syndrome and periodic limb movement disorder. Sleep 2004; 27: 557-559.

Restless Legs Syndrome Task Force of the Standards of Practice Committee of the American Academy of Sleep Medicine. An update on the dopaminergic treatment of restless legs syndrome and periodic limb movement disorder. Sleep 2004; 27: 560-583.

Michaud M, Paquet J, Lavigne G, Desautels A, Montplaisir J. Sleep laboratory diagnosis of restless legs syndrome. Eur Neurol 2002; 48: 108-113.

Allen RP, Dean T, Earley CJ. Effects of rest-duration, time-of-day and their interaction on periodic leg movements while awake in restless legs syndrome. Sleep Med 2005; 6: 429-434. 